\title{
Niveles de ruido durante el ordeño de lecherías con sistemas mecánicos del trópico alto colombiano y su efecto en la calidad de la leche y el bienestar animal
}

\section{Noise levels during the milking of dairies with mechanical systems in the highlands of the tropics of Colombia and its effect on milk quality and animal welfare}

\author{
Fredy Enrique García Castro ${ }^{1,2}$, Aldemar Zúñiga López, \\ Diana Catherine Flórez Castañeda ${ }^{1}$, Jaime Andrés Cubides Cárdenas ${ }^{1}$
}

\section{Resumen}

El objetivo del trabajo fue determinar los niveles de ruido durante el ordeño de vacas en sistemas de ordeño mecánico (fijo y móvil) y su relación con el bienestar animal y la calidad de la leche. Los niveles de ruido se midieron en ocho granjas ubicadas en municipios de la Sabana de Bogotá. La exposición al ruido de las vacas se midió en la sala de ordeño (RUME), durante la parada en el área antes de la sala de ordeño (RUIN) y al final del ordeño, con el equipo apagado (RUFI). Independientemente del tipo de ordeño, los ordeños fueron clasificados según el nivel de ruido como ordeño ruidoso (OR) y ordeño con ruido tolerable (OT). Los grupos se compararon en relación con la producción, composición y calidad de la leche, así como con los comportamientos asociados al estrés. Se encontraron diferencias significativas para el recuento de células somáticas y el número de bacterias mesófilas $(\mathrm{p}<0.05)$ siendo mayor en los ordeños denominados ruidosos.

Palabras clave: ruido; bienestar animal; bovinos de lechería; ordeño mecánico

\section{AbSTRaCT}

The objective of this study was to determine the noise levels during milking in mechanical milking systems (fixed and mobile) and their relationship with animal welfare and milk quality. The noise levels were measured in eight farms located in the municipalities of La Sabana de Bogotá (Colombia). The exposure of cows to noise was measured in the milking parlour (RUME), in the milking parlour before the milking (RUIN) and at the end of milking, with the equipment turned off(RUFI). Milking was also classified according to

\footnotetext{
${ }^{1}$ Grupo de investigación e innovación en salud y bienestar animal, (Agrosavia), Laboratorio de Salud Animal, Centro de Investigación, Tibaitatá, Bogotá, Colombia

${ }^{2}$ E-mail:fgarciac@agrosavia.co
} 
the noise level as noisy milking and milking with tolerable noise. The groups were compared in relation to the production, composition and quality of milk, as well as behavior associated with stress. Significant differences were found for the somatic cell count and the number of mesophilic bacteria $(\mathrm{p}<0.05)$, being higher in the so-called noisy milking.

Key words: noise; animal welfare; dairy cattle; mechanical milking

\section{INTRODUCCIÓN}

Los sistemas productivos ganaderos en el mundo se enfrentan a una serie de retos donde se hace necesario mejorar la productividad y la rentabilidad, pero sin olvidar las demandas de los consumidores, tales como la disminución de las emisiones de gases de efecto invernadero, el eficiente manejo del recurso hídrico, el uso racional del suelo, la integración con la biodiversidad de los ecosistemas aledaños y el bienestar a los animales. Este último reto ha tenido importantes avances en las últimas décadas y varios países han realizado esfuerzos para crear herramientas y protocolos para su adecuada evaluación, de modo que permita mejorar las prácticas tradicionales hacia una ganadería más amigable con los animales.

Dentro del contexto del bienestar animal, se conoce internacionalmente el principio de las cinco libertades como uno de los pilares para el desarrollo de esta ciencia; al menos, en cuanto a los animales domésticos se refiere (FAWC, 1992). Dentro de estas libertades se establece que se debe evitar el miedo y estrés de los animales. Para poder tener los animales en esta condición, se debe profundizar y conocer los factores que pueden generar miedo o estrés en ellos. En ese sentido, los ganaderos desconocen algunas prácticas o factores como el ruido, que son usuales en el sistema productivo, y que podrían llegar a afectar a los animales y a su productividad (Arnold et al., 2008, Brouèek, 2014).
El ruido puede definirse como sonido no deseado, ya sea continuo o intermitente, de una variedad de fuentes en el entorno. El ruido se puede considerar como un factor estresante si ocurre donde se encuentran los animales, afectando su comportamiento y niveles productivos o si induce cambios fisiológicos (Ames y Arehart, 1972). Específicamente, en la ganadería de leche se han realizado algunos ensayos para evaluar el efecto del ruido en la respuesta comportamental de los animales encontrando que las novillas expuestas al ruido de las instalaciones comerciales de ordeño muestran comportamientos de escape que se consideran como una respuesta de miedo (Arnold et al., 2007). Sin embargo, los estudios en búsqueda de la relación entre el ruido y la productividad; además de la calidad de leche, son muy limitados. Head et al. (1993) demuestran que aunque no hay cambios significativos en el volumen de leche producida, si pudiera estar influenciando el comportamiento de los animales, afectando así su bienestar.

El presente trabajo pretendió determinar el efecto del ruido sobre el bienestar de los animales, evaluando la salud de la ubre y los comportamientos asociados a la incomodidad (discomfort), así como su efecto sobre la composición de la leche, comparando dos sistemas de ordeño mecánico predominantes en los sistemas productivos de lechería especializada en la Sabana de Bogotá. 


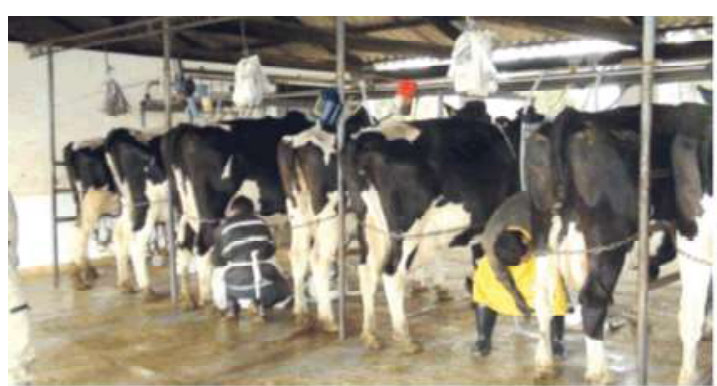

(a)

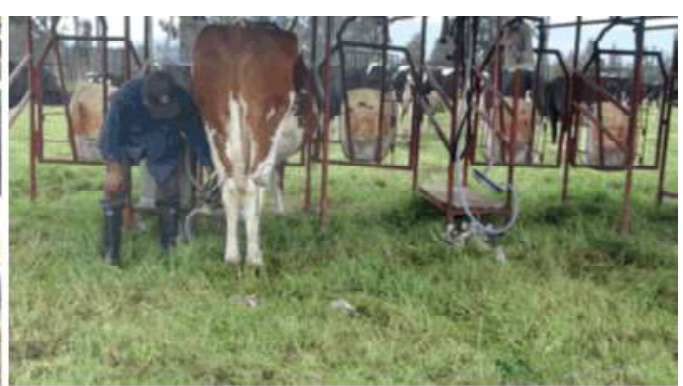

(b)

Figura 1. Sistemas de ordeño mecánico fijo (a) y mecánico móvil (b) en granjas lecheras en la Sabana de Bogotá, Colombia

\section{Materiales y Métodos}

\section{Lugar de Estudio y Animales}

El estudio se realizó en ocho ganaderías lecheras, donde cuatro de ellos usaban ordeño mecánico fijo y los otros cuatro usaban ordeño mecánico móvil (Figura 1). Los predios estaban ubicados en los municipios de Sibate, Mosquera, Madrid, El Rosal y Subachoque, todos ellos en la Sabana de Bogotá (Colombia). Los predios fueron seleccionados por tener manejo y rutinas de ordeño similares. En cada uno de los predios se realizaron cuatro visitas por mes durante el periodo de estudio (abril-julio 2014).

La raza predominante en los predios era Holstein-Friesian. Los animales usados durante el monitoreo cumplían con los siguientes criterios de inclusión: clínicamente sanos, sin historial previa de mastitis subclínica, entre segunda y tercera lactancia, dóciles en la rutina de ordeño y representativos del promedio de producción de leche en el sistema productivo.

\section{Registro del Ruido Ambiental}

En cada visita se registró el sonido en tres momentos del ordeño de las vacas. Se registró una emisión inicial durante la parada en el área antes de la sala de recolección de leche (RUIN), una emisión durante el ordeño (RUME) y otra al final del ordeño, con el equipo apagado (RUFI). Para el registro de las emisiones se utilizó el equipo Extech 407750 de respuesta rápida con un rango de registro entre 50 a $100 \mathrm{~dB}$ y para ajuste de frecuencia entre $31.5 \mathrm{~Hz}$ a $8 \mathrm{KHz}$, aunque se tomaron en cuenta los ruidos con frecuencias audibles por los bovinos que son las más altas.

\section{Muestras y Datos}

Siendo que el número promedio de animales por predio era de 26 animales, se usaron cálculos de poder basados en el estudio de García (2012) para conocer la muestra mínima con el fin de tener un cambio del $20 \%$ con un poder estadístico del $80 \%$ (Harrison y Brady, 2004), de allí que se realizó el seguimiento de 10 animales por predio $(n=80)$. En cada visita se registró la productividad (litros/ $\mathrm{vaca} /$ día) y se registraron los comportamientos de incomodidad a través de videograbación y análisis posterior con el software Cowlog 2.0. Los datos fueron contabilizados como se describe en la literatura como pasos leves (Steps/min - C1), levantamiento pronunciado del miembro (Lift/min - C2) y una patada fuerte de rechazo (Kick/min - C3) (Wenzel et al., 2003). 
Cuadro 1. Resultados descriptivos de los tres registros sonoros al inicio, mitad y final del ordeño (RUIN, RUME y RUFI, respectivamente) en ocho ganaderías lecheras de la Sabana de Bogotá (Colombia)

\begin{tabular}{lccc}
\hline & $\begin{array}{c}\text { RUIN } \\
(\mathrm{dB})\end{array}$ & $\begin{array}{c}\text { RUME } \\
(\mathrm{dB})\end{array}$ & $\begin{array}{c}\text { RUFI } \\
(\mathrm{dB})\end{array}$ \\
\hline Promedio & $70.47^{\mathrm{a}}$ & $69.84^{\mathrm{a}}$ & $71.49^{\mathrm{a}}$ \\
Desviación & 6.69 & 6.73 & 5.58 \\
estándar & 26.9 & 27.2 & 21.6 \\
Rango & 62 & 57.2 & 60.2 \\
Mínimo & 88.9 & 84.4 & 81.8 \\
Máximo & 88 & \multicolumn{3}{c}{ dentro de filas } \\
Superíndices & similares dentiva \\
indican falta de diferencia significativa
\end{tabular}

Cuadro 2. Valores promedio (desviación estándar) de los sonidos registrados al inicio, mitad y final del ordeño (RUIN, RUME y RUFI, respectivamente) en ocho ganaderías lecheras de la Sabana de Bogotá (Colombia), según el tipo de ordeño

\begin{tabular}{lccc}
\hline & $\begin{array}{c}\text { RUIN } \\
{[\mathrm{dB}]}\end{array}$ & $\begin{array}{c}\text { RUME } \\
{[\mathrm{dB}]}\end{array}$ & $\begin{array}{c}\text { RUFI } \\
{[\mathrm{dB}]}\end{array}$ \\
\hline \multirow{2}{*}{ Fija } & $67.80^{\mathrm{a}}$ & $67.63^{\mathrm{a}}$ & $68.65^{\mathrm{a}}$ \\
& $(4.2)$ & $(6.2)$ & $(4.9)$ \\
\multirow{2}{*}{ Móvil } & $73.13^{\mathrm{b}}$ & $72.05^{\mathrm{a}}$ & $74.33^{\mathrm{b}}$ \\
& $(7.3)$ & $(6.2)$ & $(4.4)$ \\
\hline
\end{tabular}

Superíndices diferentes dentro de columnas indican diferencia significativa $(p<0.05)$

Adicionalmente, se tomó una muestra individual de leche (promedio de 15 1/vaca/día) para el análisis de recuento de células somáticas (RCS/ml, Fossomatic 6 FC), composición de la leche (porcentaje de grasa, de proteína y de solidos totales). Así mismo, se determinó la calidad microbiológica de la ubre con los indicadores de recuentos de coliformes totales por medio de la técnica de recuento en placa (AOAC 989.10) y mesófilos por simetría de flujo (Lactoscan FC200, UFC/ml).

\section{Análisis de los Datos}

Se realizó estadística descriptiva y se comprobó la normalidad de las variables a través del test de Kolgomorov-Smirnov. Fue necesaria la transformación de la variable RCS a logaritmo natural para incluirla con los análisis.

Se compararon los registros de los sonidos de las tres mediciones en los dos tipos de sistemas de ordeño por medio de la prueba de T-student para muestras relacionadas. Para realizar las comparaciones de las variables productivas y de calidad de leche se crearon dos grupos denominados ordeño con sonido tolerable (OT) y ordeño con sonido ruidoso (OR), según el promedio de los tres sonidos registrados y usando $70 \mathrm{~dB}$ como valor de corte entre sonido tolerable y ruidoso, según lo planteado por Nosal et al. (2004), quienes explican que a partir de este nivel se comienza a tener efectos negativos sobre los animales.

Se usó el diseño completamente al azar factorial con medidas repetidas en el tiempo, siendo la unidad experimental los equipos de ordeño (sujetos), las visitas se hicieron a las mismas fincas (repeticiones en el tiempo) y los factores fijos (tipo de ordeño y ordeño tolerable/ruidoso). Los análisis se realizaron con el paquete estadístico SPSS v. 20.

\section{Resultados}

Los resultados descriptivos de los tres registros de sonido se encuentran resumidos en el Cuadro 1. El registro máximo de sonido fue de $88.9 \mathrm{~dB}$, el cual supera el rango máximo aceptado para bovinos. En promedio, los tres ruidos oscilaron entre 69.84 y $71.49 \mathrm{~dB}$, sin diferencias significativas entre registros. 
Cuadro 3. Valores promedios de la calidad y composición de la leche y del comportamiento asociado a la incomodidad (discomfort) de vacas Holstein-Friesian en ocho ganaderías lecheras de la Sabana de Bogotá (Colombia), según el tipo de ordeño

\begin{tabular}{lcccccc}
\hline & \multicolumn{3}{c}{ Ordeño ruidoso $^{1}$} & \multicolumn{3}{c}{ Ordeño con ruido tolerable ${ }^{1}$} \\
\cline { 2 - 7 } & Fija & Móvil & Total & Fija & Móvil & Total \\
\hline Leche (1/día/vaca) & 13.53 & 13.04 & $13.20^{\mathrm{a}}$ & 16.16 & 9.86 & $13.93^{\mathrm{a}}$ \\
RCS (RCSx10 $/ \mathrm{ml})$ & 418.45 & 960.15 & $779.58^{\mathrm{b}}$ & 364.66 & 833.06 & $529.98^{\mathrm{a}}$ \\
$\begin{array}{l}\text { Bacterias mesófilas } \\
\quad(\mathrm{UFC} / \mathrm{ml})\end{array}$ & 3,616 & 127,064 & $85,914^{\mathrm{b}}$ & 2,081 & 11,571 & $5,430^{\mathrm{a}}$ \\
Coliformes & & & & & & \\
$\quad(\mathrm{UFC} / \mathrm{ml})$ & 231.8 & 2.3 & $78.5^{\mathrm{a}}$ & 54.6 & 2.9 & $36.4^{\mathrm{a}}$ \\
Grasa (\%) & 3.17 & 3.10 & $3.19^{\mathrm{a}}$ & 3.69 & 3.53 & $3.64^{\mathrm{a}}$ \\
Proteína (\%) & 3.00 & 3.15 & $3.11^{\mathrm{a}}$ & 3.13 & 3.29 & $3.18^{\mathrm{a}}$ \\
Sólidos totales $(\%)$ & 11.51 & 11.83 & $11.72^{\mathrm{a}}$ & 12.34 & 12.23 & $12.30^{\mathrm{a}}$ \\
C1 (Steps/min) & 0.245 & 0.365 & $0.325^{\mathrm{a}}$ & 0.456 & 0.205 & $0.367^{\mathrm{a}}$ \\
C2 (Lift/min) & 0.059 & 0.247 & $0.185^{\mathrm{a}}$ & 0.170 & 0.108 & $0.148^{\mathrm{a}}$ \\
C3 (Kick/min) & 0.003 & 0.055 & $0.038^{\mathrm{a}}$ & 0.027 & 0.017 & $0.024^{\mathrm{a}}$ \\
\hline
\end{tabular}

${ }^{1}$ Ruido tolerable $<70 \mathrm{~dB}>$ ruidoso

Superíndices diferentes dentro de filas indican diferencia significativa $(p<0.05)$

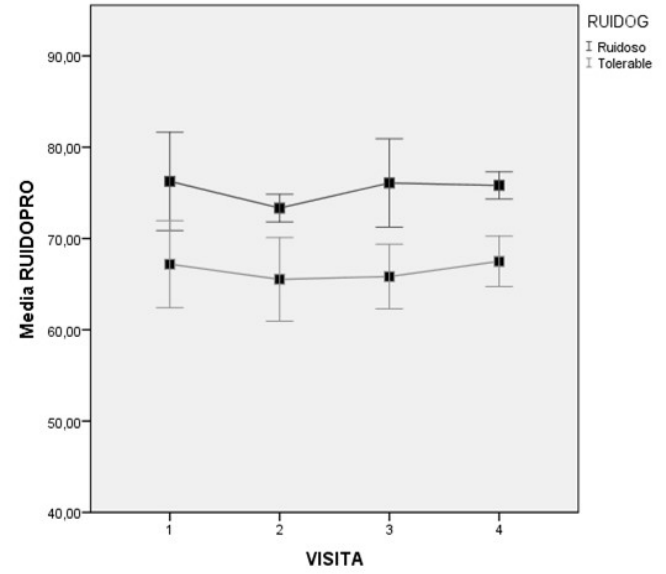

Figura 2. Ruido promedio (DE) en los ordeños ruidosos $(>70 \mathrm{~dB})$ y tolerables $(<70 \mathrm{~dB})$ en ocho ganaderías lecheras de la Sabana de Bogotá (Colombia) durante las cuatro visitas de estudio

Se encontraron diferencias significativas entre los tipos de ordeño $(\mathrm{p}<0.05)$ para los valores promedio de los registros RUIN y RUFI, donde el promedio de ruido al inicio del ordeño fue de $67.80 \mathrm{~dB}$ en el sistema fijo y de $73.13 \mathrm{~dB}$ en el sistema móvil. En forma similar, los promedios del ruido al final del ordeño fueron de 68.65 y $74.33 \mathrm{~dB}$ para los sistemas de ordeño fijo y móvil respectivamente $(\mathrm{p}<0.05)$ (Cuadro 2). El ruido a la mitad del ordeño (RUME) fue similar para ambos sistemas de ordeño. Al realizarse el análisis de los dos grupos (ruidoso vs. tolerable) se encontró que tuvieron una variación entre las visitas en un rango de 73.33 a $76.25 \mathrm{~dB}$ para los ordeños con sonido ruidoso y de 65.52 a $67.49 \mathrm{~dB}(\mathrm{p}<0.05)$ (Figura 2).

En el Cuadro 3 se muestran las variables en estudio para el tipo de ordeño según la emisión de ruido registrada. La producción de leche no estuvo afectada por el nivel ruido en el análisis global; sin embargo, en la calidad microbiológica se encontró que el conteo de células somáticas y bacterias mesófilas fueron mayores en los ordeños ruidosos (779.58 RCS x $10^{3} / \mathrm{ml}$ y $85914 \mathrm{UFC} / \mathrm{ml}$, respectivamente) respecto a los ordeños con 
ruido tolerable ( $529.98 \mathrm{RCS} \mathrm{x} 10^{3} / \mathrm{ml}$ y 5430 $\mathrm{UFC} / \mathrm{ml}$, respectivamente). Por otro lado, la composición de la leche no presentó diferencias significativas por efecto del ruido durante el ordeño.

\section{Discusión}

La industria pecuaria ha desarrollado diversos tipos de instalaciones para el manejo y para el resguardo de los animales. No obstante, algunas de estas instalaciones pueden favorecer la ocurrencia de sonidos y condiciones que no son naturales para los animales. En este estudio se evaluó el tipo de ordeño donde las instalaciones para el ordeño fijo contienen una mejor infraestructura (sala con paredes y techo), mientras los sistemas móviles se caracterizan por estar usualmente en la intemperie. El sistema móvil produjo mayor ruido al inicio y al final del ordeño, mayormente por el tipo de motores utilizados en este tipo de sistemas. Existen pocos estudios que evalúen el ruido en diferentes momentos o tipos de ordeño.

Pšenka et al. (2016), en la República Checa, estudiaron los ordeños de tipo mecánico y automático, ambos en condiciones fijas, encontrando que los animales estaban más expuestos al ruido $(72.50 \mathrm{~dB})$ con el ordeño mecánico fijo, siendo este valor cercano al encontrado en este estudio en el ordeño mecánico móvil. Asimismo, Šístková et al. (2016) reportan niveles de ruido para tres tipos de sistema de ordeño mecánico fijo (espina, rotacional y en bloque) donde los valores fueron mayores en el sistema rotacional, con un promedio de $71.2 \pm 2.56 \mathrm{~dB}$. Los resultados del presente estudio son pioneros en Colombia, donde el sistema mecánico móvil se encuentra con mayor frecuencia en los sistemas de producción de leche del trópico alto colombiano, debido a la menor inversión que se requiere.

La productividad de los animales (litros/ día/animal) no se vio afectada cuando se com pararon los grupos OR y OT, cuyo promedio cercano a los 13 1/vaca/día es representativo de la zona de estudio y evidencia la baja productividad de los pequeños productores. En este sentido, Head et al. (1993) trabajaron bajo condiciones experimentales con un grupo de animales expuestos a la grabación del ruido aeronáutico de un jet ( $115 \mathrm{~dB}$ máximo) encontrando que la producción en términos de litros de leche fue similar a la producida por los animales control (con solo ruido ambiental).

Los niveles productivos de los animales se pueden ver afectados por el ruido, debido a que los sistemas neuronales $y$ neuroendocrinos al estar afectados comprometen la eficiencia de la alimentación. Cwynar y Kolacz (2011) encontraron que ruidos de 75, 85 y $95 \mathrm{~dB}$ con frecuencias de $2 \mathrm{kHz}$ contribuyen a la reducción del apetito de los animales. Adicionalmente, Algers y Jensen (1991) encontraron una reducción de la producción de leche en vacas expuestas dos veces al día a niveles de ruido entre 80 $100 \mathrm{~dB}$ por más de una hora. Cabe resaltar que un ruido repentino de $105 \mathrm{~dB}$ podría disminuir la cantidad de leche en el próximo ordeño (Algers et al., 1978).

La composición de la leche (grasa, proteína y sólidos totales) no estuvo comprometida en este estudio, posiblemente porque los cambios en estos indicadores están mayormente asociados con la dieta de los animales.

Los mayores conteos de células somáticas y de bacterias mesófilas se presentaron en los ordeños considerados como ruidosos $(\mathrm{p}<0.05)$, sin importar el tipo de ordeño (fijo o móvil) (Cuadro 3). El efecto que puede tener el ruido sobre la salud de la ubre puede potenciarse con la constante exposición de los animales a fuentes de ruido elevado, debido a un estrés fisiológico. Se ha comprobado que las respuestas fisiológicas que suceden por el ruido, por ejemplo el sonido proveniente del motor de un tractor (97 
dB), aumenta significativamente la concentración de glucosa y los recuentos de leucocitos y reduce el nivel de hemoglobina en la sangre (Broucek et al., 1983).

Si bien el ruido puede tener una asociación directa con el aumento de las células somáticas, Gygax y Nosal (2006) encontraron, además, que la vibración durante el proceso de ordeño puede llegar a incidir en los indicadores de salud de la ubre. Estos autores encontraron una relación directa en el aumento de las células somáticas y la vibración de $0.35 \mathrm{~m} / \mathrm{s}^{2}$. En otro trabajo, Nosal y Bilgery (2004 ) asociaron el efecto de la vibración con el desajuste del sistema de vacío de los sistemas de ordeño automáticos, lo cual permitiría tener una explicación entre el ruido, la vibración y la sanidad de la ubre desde la mecánica del equipo de ordeño. Por otro lado, Kauke y Savary (2010) no encontraron efecto en los recuentos de células somáticas al evaluar el efecto del ruido (ruido alto y baja vibración; ruido bajo y alta vibración; ruido alto y alta vibración; control), mientras que Harmon (1994) mencionan que factores estresantes como el ruido pueden desencadenar la presentación de mastitis en las vacas adultas. Los resultados del presente estudio evidencian por primera vez en Colombia la asociación de ruidos moderados durante el ordeño con cambios en los recuentos de células somáticas.

Por último, en un estudio se midió el movimiento rápido de huida de las vacas al ser expuestas al ruido, donde el movimiento más rápido persistió durante los primeros cuatro días de la fase de tratamiento, con cierta evidencia de habituación de esta respuesta en el quinto día (Waynert et al., 1999). Las respuestas al ruido en instalaciones comerciales de ordeño pueden verse influenciadas por procesos de habituación. Si se considera que las vacas lecheras están regularmente expuestas al ambiente de ordeño, existe la oportunidad de reducir cualquier respuesta de miedo que surja de la exposición al ruido.

\section{Conclusiones}

- Los tipos de ordeño mecánico fijo y móvil presentaron diferencias en ruido, especialmente al inicio y final del ordeño, asociado posiblemente al funcionamiento de los equipos y la rutina de ordeño, donde el ordeño de tipo mecánico móvil presentó mayores niveles de ruido.

- El ruido afectó la composición microbiológica de la leche, en términos de altos recuentos de células somáticas.

- No se encontraron efectos del ruido sobre el comportamiento de las vacas durante el ordeño (movimientos de las extremidades).

\section{Literatura Citada}

1. Alger B, Eke SI, Stromber G 1978. The impact of continuous noise on animal health. Acta Vet Scand 67: 1-26.

2. Alger B, Jensen P. 1991. Teat stimulation and milk production during early lactation in sows: effects of continuous noise. Can J Anim Sci 71: 51-60.

3. Ames DR, Arehart LA. 1972. Physiological response of lambs to auditory stimuli. J Anim Sci 34: 994-998. doi: $10.2527 /$ jas $1972.346994 \mathrm{x}$

4. Arnold NA, Jongman KT, Hemsworth PH. 2007. The behavioural and physiological responses of dairy heifers to tape-recorded milking facility noise with and without a pre-treatment adaptation phase. Appl Anim Behav Sci 106: 12-25. doi: 10.1016/j.applanim.2006.07.004

5. Arnold NA, Kim TN, Jongman EC, Hemsworth PH. 2008. Avoidance of tape-recorded milking facility noise by dairy heifers in a Y maze choice task. Appl Anim Behav Sci 109: 201-210. doi: 10.1016/j.applanim.2007.02.002 
6. Brouèek J, Kovalcikova M, Kovalcik K.1983. The effect of noise on the biochemical characteristics of blood in dairy cows. Zivocisna Vyroba 28: 261-267.

7. Brouèek J. 2014. Effect of noise on performance, stress, and behaviour of animals. Slovak J Anim Sci 47: 111-123.

8. Cwynar P, Kolacz R. 2011. The effect of sound emission on sheep welfare. In: Proc XV International Congress of the International Society for Animal Hygiene, Vienna.

9. [FAWC] Farm Animal Welfare Council. 1992. FAWC updates the five freedoms. Vet Rec 17: 357.

10. García FE, Márquez D, Donado P, Medrano C. 2012. Bienestar animal en ganado de leche de la Sabana de Bogotá - Colombia. Bogotá, Colombia: Corpoica. $114 \mathrm{p}$.

11. Gygax L, Nosal D. 2006. Contribution of vibration and noise during milking to the somatic cell count of milk. J Dairy Sci 89: 2499-2502. doi: $10.3168 /$ jds.S0022-0302(06)72324-4

12. Harmon RJ. 1994. Physiology of mastitis and factors affecting somatic cell counts. J Dairy Sci 77: 2103-2112. doi: 10.3168/jds.S0022-0302(94)77153-8

13. Harrison DA, Brady AR. 2004. Sample size and power calculations using the non central t-distribution. Stata J 4: 142-153.

14. Head HH, Kull RC, Campos MS, Bachman KC, Wilcox CJ, Cline LL, Hayen MJ. 1993. Milk yield, milk composition, and behavior of Holstein cows in response to jet aircraft noise before milking. J Dairy Sci 76: 15581567. doi: 10.3168/jds.S0022-0302(93)77489-5
15. Kauke M, Savary P. 2010. Lärm und vibrationen im Melkstand - Auswirkungen auf das Tier. Agrarforsch Schweiz 3: 96-101

16. Nosal D, Rutishauser R, Bilgery E, Oertle A. 2004. Lärm und Vibrationen als Stressfaktoren beim Melken. FATBerichte 625: 1-12

17. Nosal D, Bilgery E. 2004. Airborne noise, structure-borne sound (vibration) and vacuum stability of milking systems. Czech J Anim Sci 49: 226-230.

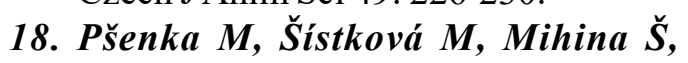
Gálik R. 2016. Frequency analysis of noise exposure of dairy cows in the process of milking. Res Agr Eng 62: 185189. doi: 10.17221/4/2015-RAE

19. Šístková M, Pšenka M, Celjak I, Bartoš P, Mihina Š, Pavlík I. 2016. Noise emissions in milking parlours with various construction solutions. Acta Technol Agric 2: 49-51. doi: 10.1515/ata2016-0011

20. Schwartzkopf-Genswein KS, Stookey JM, Welford R. 1997. Behavior of cattle during hot-iron and freeze branding and the effects on subsequent handling ease. J Anim Sci 75: 2064-2072.

21. Wayner T, Stooke DE, SchwartzkopGenswein KS, Watts JM, Waltz CS. 1999. Response of beef cattle to noise during handling. Appl Anim Behav Sci 62: 27-42. doi: 10.1016/S0168-1591(98)00211-1

22. Wenzel CS, Schönreiter-Fischer S, Unshelm J. 2003. Studies on step-kick behavior and stress of cows during milking in an automatic milking system. Livest Prod Sci 83: 237-246. doi: 10.1016/ S0301-6226(03)00109-X 